\title{
An innovative nurse-led cystoscopy clinic in Denmark
}

\author{
Susanne Quallich PhD, ANP-BC, NP-C, CUNP, FAANP ${ }^{1}$ ๑ | Anders Frey MD $^{2}$ | \\ Peter Busch Østergren $M^{3}$ | Søren Sorensen Madsen MD ${ }^{2}$
}

${ }^{1}$ Department of Urology, Michigan Medicine, University of Michigan, 3875 Taubman Center, Ann Arbor, Michigan

${ }^{2}$ The Department of Urology, The University Hospital of Southwestern Denmark, Esbjerg, Denmark

${ }^{3}$ Department of Urology, Herlev and Gentofte Hospital, Herlev, Denmark

\section{Correspondence}

Susanne A. Quallich, Department of Urology, Michigan Medicine, University of Michigan,

3875 Taubman Center, 1500 E. Medical

Center Drive, SPC 5330, Ann Arbor, MI

48109-5330.

Email: quallich@umich.edu
The need for serial surveillance cystoscopy after a diagnosis of bladder cancer and long wait times led to the development of a nurse-led cystoscopy in The University Hospital of Southwestern Denmark, in Esbjerg, Denmark. This paper discusses the background, development and success of these training programme in Esbjerg, Denmark. Four nurses have participated in a structured in-house training programme that started in 2014. In the last 4 years these four nurses have successfully performed 1024 cystoscopies. To date, there have not been any poor outcomes, in terms of missed diagnosis or procedure complications. This programme has offered a way to improve satisfaction of the residents and staff urologists while successfully and safely meeting the needs of the community.

\section{KEYWORDS}

advanced nurse practitioners, advanced practice, bladder cancer, nurse cystoscopist, surveillance

\section{1 | INTRODUCTION AND BACKGROUND}

Denmark has an estimated annual rate of new bladder cancer diagnoses of 1700 per year, ${ }^{1,2}$ a rate that has remained relatively stable. Many of these men and women will require surveillance cystoscopy as frequently as every 3 to 6 months for low grade or intermediate grade bladder tumours, as recommended by the current European Association of Urology guidelines. ${ }^{3}$ However, meeting the demands of this surveillance schedule has been a challenge, especially in the more rural areas of Denmark, and this is also limited by the availability of urologists to perform the surveillance cystoscopies.

Globally, there are examples of successful nurse-led cystoscopy clinics. In Australia, this arose out of necessity, due to the volume of Australians needing cystoscopy and the inability of urologists alone to meet the need. Nurse practitioners in Australia are restricted to surveillance cystoscopy by their national scope of practice. However, Chatterton et al. ${ }^{4}$ reported a $72 \%$ reduction in wait times for surveillance cystoscopy for bladder cancer patients during the study period. Sapre et al. $^{5}$ and Chatterton et al. ${ }^{4}$ additionally describe improved continuity of care, as the nurse practitioners are able to perform surveillance cystoscopy after administering intravesical therapy.

A similar scenario drove the development of the UK programme to train nurse cystoscopists. ${ }^{6,7}$ Nurse-cystoscopists in the United
Kingdom are trained and certified under standards established by the British Association of Urologic Surgeons (BAUS). This has allowed for more timely access to surveillance flexible cystoscopy clinics, helping to address the issue of long wait times. This initiative has been successful, and anecdotal reports suggest that nearly $50 \%$ of UK urologists now utilize nurse-cystoscopists. ${ }^{7}$ Gidlow et al. ${ }^{8}$ reported that agreement between the findings between a specialist urology nurse and an urologist in the United Kingdom was kappa value (к) of 0.95 . Taylor et al. ${ }^{9}$ found that the sensitivity and specificity of trained nurse-cystoscopists for surveillance cystoscopy findings were $96 \%$ and $100 \%$, respectively, with a kappa value of 0.94 . Diagnostic cystoscopies executed by nurse-cystoscopists were not significantly different, with respect to findings or the treatment plans developed, compared to those of urologist-executed cystoscopies. ${ }^{9}$ Nurse cystoscopists were more likely to refer UK patients for cystoscopy under general anaesthesia, leading to increased costs when compared with urologists; this decreased over time, though statistical significance persisted. ${ }^{10}$ Neither Taylor et al. ${ }^{9}$ nor Gidlow et al. ${ }^{8}$ reported any difference in the referral patterns for general anaesthesia of nurse cystoscopists compared to urologists, however.

The University Hospital of Southwestern Denmark is a 370-bed hospital in Esbjerg, Denmark, and is 200 miles southwest of Copenhagen. The hospital's surrounding area has a population of 
approximately 250000 , and it is a more rural area of Denmark with more limited access to specialty services. This access pressure offered reason to develop a nurse cystoscopy training programme and eventual nurse-led cystoscopy clinics. Against this backdrop we aimed to discuss the development and success of this training programme in Esbjerg, Denmark.

\section{DEVELOPMENT OF THE NURSE-LED CYSTOSCOPY CLINIC}

The nurse-led cystoscopy programme was originally conceived from the recognition that maintaining the cystoscopy surveillance schedule for patients diagnosed with pTa low-grade bladder cancer was a challenge in this rural area of Denmark. Its rural location presented problems with staffing, both in the form of challenges recruiting residents to this this area, as well as challenges recruiting trained urologists. But the development of this programme was accompanied by motivation from the existing clinic nurses to expand their practice and learn office cystoscopy procedures, to provide some continuity of care for patients. Waiting times had only a minor influence, but were also a recognized issue driving the establishment of this training. In addition to low-grade bladder cancer follow-up, nurses also learned to remove double-J ureteral catheters via cystoscope as part of the initial training programme.

\section{1 | Education program}

The training of nurses began at the 1 November 2014, and the three nurses included in this programme were nurses with both substantial experience in urology and in assisting urologists with flexible office cystoscopy. All nurses had 1 day of theory and didactic content that included specifics of bladder cancer, benign bladder polyps and other pathological findings in the bladder (Table 1). Furthermore, they had theoretical introduction to cystoscopy techniques and practiced manipulation and use of the cystoscope on inanimate models (Table 2). This day of education was designed and conducted by Søren Madsen (SM), a chief physician and urologist at The University

TABLE 1 Example basic didactic content to prepare nurses for cystoscopy

- Gather focused GU history, including risk factors for lower urinary tract pathology

- Define the indications, including clinical signs and symptoms, for in-office cystoscopy

- Exhibit thorough understanding of the contraindications, individual risk factors and benefit-risk considerations for an individual patient

- Define pre-procedural information and expectations to be discussed with the patient

- Describe the normal anatomy, histology, and cystoscopic findings of the lower urinary tract

- Discuss common benign and malignant pathology of the lower urinary tract, including anatomical variants

- Describe the potential complications during ureteral stent removal and the appropriate actions in the event of these complications

- Explain management principles of post-procedural urinary infection, urosepsis, haematuria, anaphylaxis and proper management of these conditions

\section{WHAT IS KNOWN ABOUT THIS TOPIC}

- There is scare literature that documents the expansion of nurses' role in Denmark, and the progress towards more "advanced practice".

- This paper contributes evidence of the evolving role of expert nurses in the urology clinical environment.

- Continued nursing role expansion in Denmark reflects global recognition for the nursing role, and will mirror previous nursing role expansion seen in Danish hospitals and across Europe.

\section{WHAT THIS PAPER ADDS}

- Expansion of the role of nursing can contribute to Denmark's national goals for care to shift to the outpatient arena. This paper offers evidence that there is ongoing nursing role expansion within urology clinical environments, as demonstrated by the specialty training in performing cystoscopy received by this small group of nurses.

- This continued expansion of the nursing role in Denmark (Sygeplejersker) offers the opportunity for improved adherence to surveillance guidelines for targeted chronic urology conditions.

- Role expansion represents the capacity of Danish nurses to stand among their global nursing peer group as expert nurses, with the opportunity to contribute to the care of their country's citizens, a position strongly advocated for nurses by the World Health Organization.

Hospital, with his career based on treating bladder cancer as support for the programme development. No specific research or teaching protocol was developed prior to document the initiation of the nurseled flexible cystoscopy programme.

After the day of theory education, nurses were then allowed to perform supervised cystoscopies; initially nurses were directly

TABLE 2 Basic skills to prepare nurses for cystoscopy

- Understand proper patient positioning

- Explain preparation of equipment and environment for cystoscopy procedures

- Define the function and performance characteristics of the equipment to be used during the procedure, including video monitor and illumination equipment, and their capabilities and limitations

- Demonstrate the ability to recognize equipment malfunction

- Prepare and introduce the cystoscope into the urethra

- Demonstrate the safe use of the deflection, inflection, and scope rotation systematically examine bladder mucosa and identify bladder landmarks

- Demonstrate safe grasp and removal of ureteral stents

- Documentation of findings 
supervised for a month. S.M. initially supervised the cystoscopies directly by being present in the room during the entire procedure. If the nurse was found to have sufficient skill and experience, they were permitted to perform the cystoscopies without direct supervision, but with S.M. being on call if any questions arose. After the first month, nurses cleared by S.M. were allowed to perform the cystoscopies independently. The nurse-cystoscopists were evaluated based on skill and proficiency under supervision, rather than focusing on a specific number of cystoscopies that needed to be completed. If anything suspected of being malignant or pathologic was found during the nurse-performed cystoscopy, a physician would be called to confirm a diagnosis.

The first follow-up cystoscopy after transurethral resection of bladder tumour (TURBT) patients is performed by a physician; the patient is informed at that time that the next visit will be with a specially-trained nurse. Hereafter the nurses themselves collected informed consent for the procedure, performed the cystoscopy and cleaned the scopes in the clinic with the help of service workers. If a lesion is found by the nurse that requires intervention, the nurse notifies a resident, who prepares the patient for surgery.

Presently, four nurses are performing flexible office cystoscopies, and cystoscopies are performed in the ambulatory clinic for the full 5 days every week. No specific ongoing evaluation of the four nurses is being performed; however, daily supervision ensures that the high standards are maintained. The number of patients seen each week is 20 patients on average (men and women), divided among the four nurses. Two nurses are assigned to perform cystoscopies each ambulatory clinic day. No patient with suspect cystoscopy findings leaves the hospital without having seen an urologist, and the nurse can also ask a resident or an urologist to supervise the cystoscopy procedure as needed. When the nurses had gained experience in basic flexible cystoscopy, the nurses were also taught to remove double-J stents and perform cold cup biopsies with local anaesthesia. To date, no procedure complications have been reported and no cystoscopic examinations have had to be repeated. This is a consequence of the nurses being extremely thorough during their cystoscopic examination, and the urologists being at hand and willing to supervise when needed.

At first the nurses only performed cystoscopy on patients with $\mathrm{Ta}$ lesions (low-grade bladder tumour), and patients who had at least one previous cystoscopy by a urology resident or consultant. Since the initiation of the project, and up to August 2018, the four trained nurses have performed 1024 cystoscopies of which 161 have included biopsies (Table 3). It is anticipated that the nurse-cystoscopists would supervise the education of other nurses, with the training based on the programme developed by S.M., if the need for more cystoscopy

TABLE 3 Number of nurse-led cystoscopies November 2014 to August 2018

\begin{tabular}{|lll|} 
& Cystoscopies & Cystoscopy + biopsy \\
\hline Nurse 1 & 402 & 52 \\
\hline Nurse 2 & 363 & 70 \\
\hline Nurse 3 & 187 & 37 \\
\hline Nurse 4 & 72 & 2 \\
\hline Total & 1024 & 161 \\
\hline
\end{tabular}

nurses developed. In fact, one additional nurse cystoscopist has been trained in this manner.

\section{3 | OUTCOMES}

In the broader pool of urologists, a major concern has been that nurse-led flexible cystoscopy would diminish the training of urology residents. However, the experience gained at our centre has demonstrated that the volume of patients needing flexible cystoscopy have made it feasible for residents maintain their own ambulatory flexible cystoscopy days, with the opportunity to select the potentially more interesting or complex cases. Prior to this nurse-cystoscopist initiative, there were general complaints that flexible cystoscopies took up too much time for the residents, due to the volume of patients needing surveillance cystoscopy, again limiting the ability to recruit residents to this more rural site.

Our experience so far offers no evidence to suggest that other facilities could not institute a similar programme to decrease wait times by expanding nurses' clinical skills. Nurses with experience in assisting with cystoscopies and training by an urologist or experienced nurse-cystoscopist will quickly become proficient in a clinic environment that offers education and supervision. While patient satisfaction has not been formally measured, anecdotally there have been no complaints, and patients have not refused evaluation by the nursecystoscopist.

\section{1 | Next steps}

Because this nurse-led cystoscopy initiative was successful, the independent role of the nurses in our urology clinic was expanded further, as is consistent with the emerging advanced nurse practitioner (ANP) role advocated by the Danish Nurses' Organization. ${ }^{11,12}$ A nurse-led LUTS (lower urinary tract symptoms) outpatient clinic was started. A nurse initiates the patient evaluation by gathering the urine for urinalysis, performing the basic flow evaluation, and performing a flexible cystoscopy. Many patients can be referred back to their private general practitioner after this initial work up and treatment; otherwise they will be given a time with one of the urologists for additional evaluation and management. If a thorough urodynamic examination is needed, it is first authorized by a physician; this authorization would occur during a daily case review, which is scheduled in one of the senior physician clinics. There is always allocated 45 minutes of consultation time in one of the doctors' schedules when there is a nurse LUTS clinic scheduled.

\section{4 | DISCUSSION}

There are over 2500 advanced practice urology nurses across Europe offering services such as flexible cystoscopy, prostate health clinics, sexual dysfunction clinics and lower urinary tract assessment, with high rates of patient satisfaction and safety. ${ }^{13}$ The United Kingdom has had advanced practice nurse-led prostate biopsy since $2010 .{ }^{14}$ UK nurses have been performing conventional ultrasound-guided 
prostate biopsies since 2004 (Henderson et al., 2004), ${ }^{15}$ although this may change as the magnetic resonance imaging (MRI)-guided biopsy becomes a standard. UK's advanced nurses have also been leading cystoscopy clinics for several years. ${ }^{13}$ Evolving the clinic nurse role into a more ANP role with the surveillance cystoscopy clinics at The University Hospital of Southwestern Denmark evolved out of necessity, as the trained urologists could not keep up with the needs of the local community. This manner of growth of the non-physician provider workforce in urology globally, however, can help to bridge the acknowledged growing gap in access to urology care, especially as populations continue to age.

By way of comparison to this new training programme at The University Hospital of Southwestern Denmark, there is no nationally accepted training programme, accreditation or specific legal requirements for advanced practice nurses to perform prostate biopsies in the United Kingdom (or Denmark). A collaborative effort between the British Association of Urologic Nurses (BAUN) and the European Association of Urologic Nurses (EAUN) developed guidelines for best practice that informed competencies for performing prostate biopsies. Recommended preparation includes dimensions such as obtaining informed consent, documenting a comprehensive medical history, proficiency with performing digital rectal examination, proficiency in performing the biopsy procedure itself and maintenance of minimum number of procedures and ongoing knowledge each year. These guidelines can be extrapolated to other urologyspecific procedures (such as cystoscopy) that can fall under the domain of an expert nurse. The BAUN/EAUN guidelines include the ability of the advanced practice nurse to recognize complications and seek guidance from a collaborating urologist as needed. ${ }^{14}$ Shah and Baston (2013) likewise report on the success of ANP who perform prostate biopsies. ${ }^{16}$ After a regimented training period and periodic audits evaluating patient satisfaction, there was no difference in cancer detection rates or complications between urologists and ANP.

This expansion into areas of procedural care in urology to improve access for patients in the area of The Hospital of Southwestern Jutland offers support that the role of the nurse in Denmark is expanding into domains that require clinical expertise and critical thinking, collaboration, and problem solving, all of which are recognized hallmarks of advanced nursing practice. This cystoscopy training parallels the Urology Nurse Practitioner (NP) competencies ${ }^{17}$ that offer a method for advancing the clinical acumen of all nursing professionals in urology. This advancement of the nursing role is further characterized by the larger societal need of the local community for specialized care, one that this advancement in practice by these nurse cystoscopists has filled by demonstrating proficiency in three distinct domains: patient care, professionalism, and their role within the health system itself. ${ }^{12}$ The specialized nurse cystoscopists of the Hospital of Southwestern Jutland meet the criteria established by the Danish Nurses Organization ${ }^{11}$ for the role of "Advanced Nurse Practitioner", and their advanced role offers a further exemplar of this ANP role, as it is emerging in Denmark.

\section{5 | CONCLUSIONS}

Nurse-led cystoscopy clinic at The University Hospital of Southwestern Denmark has offered an option to maintain the necessary bladder cancer surveillance schedule for patients, while expanding the complexity of patients that urology residents are offered. To date, there have not been any poor outcomes, in terms of missed diagnosis or procedure complications. This programme has offered a way to improve satisfaction of the residents and staff urologists at The University Hospital of Southwestern Denmark while successfully and safely meeting the needs of the community. Furthermore, this programme offers another exemplar for advanced nursing practice in Denmark.

\section{ACKNOWLEDGEMENTS}

The authors would like to acknowledge Birgitte Tingskov, Lene Hansen, Mirjam Hansen and Vibeke Larsen, the nurse-cystoscopists that have made this program a continued success for The University Hospital of Southwestern Denmark. This paper was supported by the CopMich Collaborative, a partnership between Michigan Medicine, University of Michigan, Department of Urology and University of Copenhagen/Herlev Hospital, Department of Urology. This collaboration was established to promote excellence in urology patient care at all levels, and the exchange of ideas between the two universities.

\section{CONFLICT OF INTEREST}

The authors declare no conflict of interest.

\section{ORCID}

Susanne Quallich (D) https://orcid.org/0000-0002-6522-4035

\section{REFERENCES}

1. Antoni S, Ferlay J, Soerjomataram I, Znaor A, Jemal A, Bray F. Bladder cancer incidence and mortality: a global overview and recent trends. Eur Urol. 2017;71(1):96-108. https://doi.org/10.1016/j.eururo.2016. 06.010.

2. Bagi $P$, Nordsten $C B$, Kehlet $H$. Cystectomy for bladder cancer in Denmark during the 2006-2013 period. Dan Med J. 2016;63(4):A5217.

3. Witjes JA, Lebret T, Compérat EM, et al. Updated 2016 EAU guidelines on muscle-invasive and metastatic bladder cancer. Eur Urol. 2017;71(3):462-475. https://doi.org/10.1016/j.eururo.2016.06.020.

4. Chatterton K, Bugeja $\mathrm{P}$, Challacombe B, Anderson P, Costello A. Nurses' experience establishing a nurse-led bladder cancer surveillance flexible cystoscopy service. Lamp. 2011;68(8):37(1).

5. Sapre N, Bugeja P, Hayes E, Corcoran NM, Costello A, Anderson PD. Nurse-led flexible cystoscopy in Australia: initial experience and early results. BJU Int. 2012;110:46-50. https://doi.org/10.1111/j. 1464-410X.2012.11472.x.

6. BAUS/BAUN. Flexible Cystoscopy Training and Assessment Guideline. 2nd ed. London, England: British Association of Urological Surgeons; $2017 a$.

7. BAUS/BAUN. Flexible Cystoscopy: Performance Criteria, Training and Assessment Logbook. 2nd ed. London, England: British Association of Urological Surgeons; 2017b.

8. Gidlow A b, Laniado $M$ e, Ellis B w. The nurse cystoscopist: a feasible option. BJU Int. 2000;85(6):651-654. https://doi.org/10.1046/j.1464410x.2000.00574.x.

9. Taylor JM, Pearce I, O'Flynn K j. Nurse-led cystoscopy: the next step. BJU Int. 2002;90(1):45-46. https://doi.org/10.1046/j.1464-410X.2002. 02831.x.

10. Radhakrishnan S, Dorkin TJ, Johnson P, Menezes P, Greene D. Nurse-led flexible cystoscopy: experience from one UK Centre. BJU Int. 2006;98(2): 256-258. https://doi.org/10.1111/j.1464-410X.2006.06341.x.

11. Danish Nurses Organization. Advanced Nurse Practitioners-Improved Healthcare to the Chronically III. Denmark: Dansk Sygeplejeråd; 2008. 
12. Quallich SA, Grauengaard J, Ohl DA, Sønksen J. Review of advanced nursing practice in Denmark with an exemplar. Int J Urol Nurs. 2018; 12:96-99. https://doi.org/10.1111/ijun.12170.

13. Hori S, Fuge O, Trabucchi K, Donaldson P, McLoughlin J. Can a trained non-physician provider perform transrectal ultrasound-guided prostatic biopsies as effectively as an experienced urologist? BJU Int. 2013;111(5):739-744.

14. Turner B, Aslet P. Nurse practitioner-led prostate biopsy in the United Kingdom. Urol Nurs. 2011;31(6):351-355.

15. Henderson A, Andrich DE, Pietrasik ME, Higgins D, Montgomery B, Langley SEM. Outcome analysis and patient satisfaction following octant transrectal ultrasound-guided prostate biopsy: a prospective study comparing consultant urologist, specialist registrar and nurse practitioner in urology. Prostate cancer and prostatic diseases. 2004;7(2):122-125.
16. Shah J, Baston E. Standard of prostate biopsies undertaken by nurse practitioners. Can Nurs Prac. 2013;12:32-35.

17. Quallich SA, Bumpus SM, Lajiness S. Competencies for the nurse practitioner working with adult urology patients. Urol Nurs. 2015;35(5): 221-230. https://doi.org/10.7257/1053-816X.2015.35.5.221.

How to cite this article: Quallich S, Frey A, Østergren PB, Madsen SS. An innovative nurse-led cystoscopy clinic in Denmark. Int J Urol Nurs. 2019;13:39-43. https://doi.org/10.1111/ijun. $\underline{12181}$ 